\title{
Magnetic decoration of Escherichia coli loaded with Palladium nanoparticles
}

\author{
Ana Lucía Campaña \\ Department of Physics \\ University of Oslo \\ Oslo, Norway \\ a.1.c.perilla@fys.uio.no
}

\author{
Pavlo Mikheenko \\ Department of Physics \\ University of Oslo \\ Oslo, Norway \\ pavlo.mikheenko@fys.uio.no
}

\author{
Nadeem Joudeh \\ Department of Biosciences \\ University of Oslo \\ Oslo, Norway \\ nadeem.joudeh@ibv.uio.no
}

\author{
Dirk Linke \\ Department of Biosciences \\ University of Oslo \\ Oslo, Norway \\ dirk.linke@ibv.uio.no
}

\begin{abstract}
Palladium (Pd) is a scarce metal, which is able to catalyze a variety of important chemical reactions. In the bulk form, Pd is anomalous paramagnetic, but in the form of nanoparticles, it has been reported as ferromagnetic. Detecting the magnetism of Pd nanoparticles is, however, difficult due to their reduced dimension. In addition, the ferromagnetic nature is a size-dependent property and, for Pd, the strength of magnetism decreases with slight increments in size. A "green" methodology for the preparation of small Pd nanoparticles was followed in this study. It is based on the ability of bacteria to take up $\mathbf{P d}^{2+}$ ions from its surrounding solution and to enzymatically reduce it to metallic $\mathbf{P d}^{0}$ nanoparticles. The efficiency of the production can be determined by transmission electron microscopy and, as a new technique, magnetic force microscopy. Notwithstanding, the study of nanoparticles of the size of just a few nanometers with these techniques is still difficult. Here we present a methodology for the enhancement of the magnetic signal of biologically produced Pd-based nanoparticles through the decoration of Escherichia coli bacteria cross-sections with $\mathrm{Fe}$ nanoparticles. This methodology allows the visualization of bacteria that are loaded with magnetic nanoparticles even when conventional transmission electron microscopy has difficulties to resolve them inside the microorganisms.
\end{abstract}

Keywords-Pd nanoparticles, transmission electron microscopy, magnetic force microscopy, Escherichia coli, magnetic decoration.

\section{INTRODUCTION}

Palladium $(\mathrm{Pd})$ is a rare element in the earth crust, which is intensively used in numerous catalytic reactions [1-3]. In recent years, the unique properties of Pd nanoparticles (NPs) promoted their intensive experimental and theoretical study. The use of Pd in the form of nanoparticles offers a higher surface area for the interaction with other materials, which overall increases the performance of the element in catalysis [4-6]. Several reports have presented the size-dependent ferromagnetic nature of Pd NPs, which could expand the area of their applications [7-10]. Measuring the magnetism of $\mathrm{Pd}$ NPs is, however, not simple because their size and magnetic moment are smaller than that of traditionally used NPs, which are based on metals providing strong magnetization: $\mathrm{Fe}, \mathrm{Co}$, or Ni [11]. The cost-effective production of Pd NPs also remains a challenge. In an attempt to resolve this problem, biological routes for production of Pd NPs have been developed using a variety of biological models $[6,12,13]$ including bacteria such as Escherichia coli (E. coli) $[14,15]$. In these biological processes, the Pd NPs are found intracellularly, in the periplasmic space or extracellularly. To observe Pd NPs, transmission electron microscopy (TEM) [16-18] or magnetic force microscopy (MFM) [19,20] are used.

The application of MFM for the detection of Pd NPs is a new activity [20], which became possible after the development of scanning probe microscopes and engineering sensitive nanometer-scale magnetic probes. MFM is the main technique in this study. The visualization of the magnetism of pure Pd NPs inside or surrounding the cell is an arduous labor, therefore in this work, a modification in the sample preparation to increase their magnetic response is tested. This modification consists of the decoration of already prepared cross-sections of Pd-based NP-loaded bacteria with $10 \mathrm{~nm}$ $\mathrm{Fe}_{2} \mathrm{O}_{3} \mathrm{NPs}$ in the presence of a strong external magnetic field perpendicular to the sample. The complete description of this technique and the obtained results are the main subjects of this paper.

\section{EXPERIMENTAL}

\section{A. Bacteria growth and nanoparticle production}

Escherichia coli (E. coli) BW25113 strain was used for the production of Pd-Fe NPs. A single colony was used to inoculate $10 \mathrm{ml}$ Lysogeny Broth (LB) starter culture and incubated overnight at $37^{\circ} \mathrm{C}$ with shaking at $200 \mathrm{rpm}$. On the next day, $0.5 \mathrm{ml}$ of the starter culture was used to inoculate $50 \mathrm{ml} \mathrm{LB}$ in an Erlenmeyer flask incubated at $37^{\circ} \mathrm{C}$ with shaking at $200 \mathrm{rpm}$ until the optical density $\left(\mathrm{OD}_{600}\right)$ of the culture reached 0.5 . The cells were harvested and washed two times with $20 \mathrm{mM}$ MOPS buffer $(\mathrm{pH} 8)$ by centrifuging at $4200 \mathrm{~g}$ before normalizing the cell concentration to $\mathrm{OD}_{600}$ equal to 3.5. For nanoparticles formation, E. coli suspensions were exposed to $1 \mathrm{mM}$ sodium tetrachloropalladate (II) $\left(\mathrm{Cl}_{4} \mathrm{Na}_{2} \mathrm{Pd}\right)$ and $1 \mathrm{mM}$ iron (III) chloride $\left(\mathrm{FeCl}_{3}\right)$ (both purchased from Sigma Aldrich, Norway). After 2 hours of incubation at $30^{\circ} \mathrm{C}, 10 \mathrm{mM}$ sodium formate was added to initiate the reduction reaction to neutral $\mathrm{Pd}$ and $\mathrm{Fe}$, and the solution was left to react until the solution turned black. 


\section{B. Sample embedding}

For the embedding of the bacterial samples, the bacteria were thoroughly washed, fixed, dehydrated, and finally embedded in epoxy resin, according to Deplanche et.al [21]. The Pd-Fe NP-loaded bacteria were fixed in $4 \%$ paraformaldehyde and 2\% glutaraldehyde (v/v) (both purchased from Sigma Aldrich, Norway) dissolved in $20 \mathrm{mM}$ MOPS buffer $(\mathrm{pH} 8)$ overnight at $4^{\circ} \mathrm{C}$. The samples were washed twice with distilled water. The dehydration was performed in a series with ascending ethanol concentrations of $50 \%, 70 \%, 90 \%$ and $96 \%$ ethanol for $10 \mathrm{~min}$ each, followed by centrifugation at $5900 \mathrm{~g}$ for 2 minutes. Finally $100 \%$ ethanol was used four times for $15 \mathrm{~min}$ each. The samples were then incubated in $1 \mathrm{~mL}$ of $100 \%$ acetone for 15 min followed by an incubation in 1:1 acetone-EPON solution at room temperature overnight. The NP-loaded cells were centrifuged at $3500 \mathrm{~g}$ for $10 \mathrm{~min}$ and incubated in $100 \%$ EPON for $1 \mathrm{~h}$ at room temperature before finally drying them in an oven at $60^{\circ} \mathrm{C}$ for 3 days. The hardened resin was sliced with a Diatome ultra $45^{\circ}$ diamond knife (Diatome Ltd., Switzerland) into slices of $0.5-1 \mu \mathrm{m}$ of thickness, which were set on top of clean glass microscope coverslips and inserted into MFM for measurements. Thinner slices of the resin $(70-100 \mathrm{~nm})$ were mounted on copper grids for imaging with TEM.

\section{Transmission electron microscopy (TEM)}

TEM samples were investigated using a JEOL 1400 plus (JEOL, Japan) Electron Microscope at 60-100KV equipped with element Energy Dispersive X-Ray Analysis (EDX).

\section{Magnetic Force Microscopy (MFM)}

Magnetic force microscopy (MFM) was performed using a scanning probe microscope, NanoWizard (JPK Instruments, UK) with a 4.0 pyramidal-tipped silicon cantilever PPP-MFMR-10 coated with a magnetic cobalt film (Nanosensors, Switzerland). The samples were studied using the two-pass scanning technique [22]. In this technique, the first scan is performed close to the surface of the sample measuring its topography. In the second pass, the scanned topography is followed at a certain height, large enough to distinguish short-range (van der Waals) from longrange (e.g. magnetic) forces. The measurements were done in $\mathrm{AC}$ mode at the resonance frequency of $74 \mathrm{KHz}$. Previous studies revealed that the optimum height for the used cantilevers and Pd-Fe NPs, at which van der Waals forces could be neglected but magnetic interaction is still strong, was between 20 and $30 \mathrm{~nm}$ of lift height [20]. The magnetic signal was measured via phase shift of the cantilever oscillations, which is proportional to the gradient of force between the probe and the sample [22-25]. The NPs were magnetized by a magnet set below the slices of the strength of $0.58 \mathrm{~T}$.

\section{E. Magnetic decoration}

The deposition of $\mathrm{Fe}_{2} \mathrm{O}_{3}$ NPs on top of the resin sections was carried out to evaluate their effect as a magnetic signal enhancer for the low magnetic response of the Pd-based nanoparticles. The non-specific attachment of magnetic NPs has been used previously, including applications in separation, targeting and localization of the magnetic response of NPs [26]. A $20 \mu \mathrm{L}$ drop of a $5 \mu \mathrm{g} / \mathrm{mL}$ dilution of single core magnetic $\mathrm{Fe}_{2} \mathrm{O}_{3}$ nanoparticles with an average diameter of $10 \mathrm{~nm}$ in distilled water was spread on top of the sample. During this procedure, the sample was subjected to a $0.58 \mathrm{~T}$ external magnetic field perpendicular to the slice surface, and the drop was left to dry at room temperature for 2 hours. The samples with $\mathrm{Fe}_{2} \mathrm{O}_{3}$ NPs were later studied with MFM.

\section{F. Image processing}

The images obtained with TEM were imported into the image processing software ImageJ for analysis. To avoid uneven illumination of the images, the image threshold procedure was applied, and the NP diameter was calculated manually drawing a horizontal and perpendicular line on top of the desired NPs for a representative sample.

\section{RESULTS AND DISCUSSION}

AFM and MFM studies of bacteria commonly use fixed and dried samples on top of a smooth and clean surface to immobilize the cells or nanoparticles [27-29]. This methodology has one relevant shortcoming. It is only possible to obtain information about forces close to the surface. Therefore, the weak magnetism of the Pd NPs deeper inside the sample requires the use of different sample preparation techniques in order to improve the interaction of the tip with the NPs inside or around the bacteria. As described in previous work [19, 20], EPON embedding of bacterial samples - as is usual for TEM of biological samples - can be used for the visualization of the samples in the MFM. The samples in the form of thin resin slices allow to study the Pd or Pd-Fe NPs in different locations with respect to the bacteria that produced them, both by TEM and MFM. After the study of a sample, magnetic decoration of the slice surface with commercial $\mathrm{Fe}_{2} \mathrm{O}_{3}$ NPs was carried out. This was done in order to amplify the low magnetic signal of the small Pd-based nanoparticles inside or in contact with the bacteria which are otherwise difficult to resolve magnetically.

\section{A. TEM characterization}

The correct embedding of E. coli along with the NPs was evaluated by TEM. The cross-sections of bacteria with Pd-Fe NPs produced by them are presented in Fig. 1a. The NPs are seen as clusters of black particles in the bacterial membrane or as NP chains connecting different cells. In addition, a negative control sample was prepared without using the precursors $\mathrm{Na}_{2} \mathrm{PdCl}_{4}$ and $\mathrm{FeCl}_{3}$. An example of the TEM for the control sample is shown in Fig. 1b, in which, as expected, there is no evidence of nanoparticles inside or around the bacteria.

In spite of the thorough-washing steps of NP-loaded $E$. coli before embedding, clusters of $\mathrm{Pd}-\mathrm{Fe} \mathrm{NPs}$ remain attached to bacteria (Fig. 1c) and also clusters of pure Fe needle-like grey crystals (Fig. 1e) can be seen in the proximity of bacteria. The elemental composition of both types of clusters was confirmed by EDX analysis. As shown in Fig. 1d, the Pd-Fe NPs clusters present peaks of Pd (yellow arrow) and low peaks of $\mathrm{Fe}$ (white arrows). 


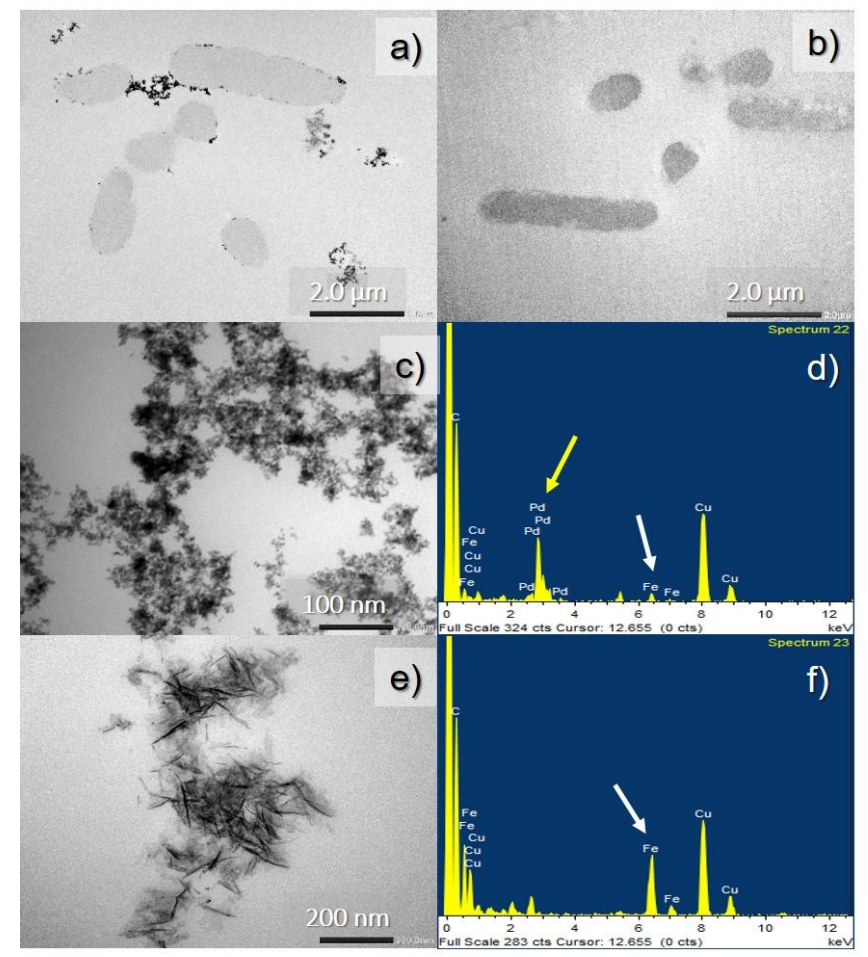

Fig. 1. TEM results for the resin slices with (a) E. coli with Pd-Fe NPs and (b) control unloaded E. coli. Close up study and EDX analysis of the clusters of Pd-Fe nanoparticles (c, d) and clusters of pure Fe needle-like grey crystals (e, f).

In contrast, the needle-like crystal structures are composed mainly of Fe, without traces of Pd (Fig. 1f). The additional $\mathrm{Cu}$ peaks come from the support surface (copper grids) [30]. A size distribution histogram was calculated for a statistically representative sample with $\mathrm{Pd}-\mathrm{Fe} \mathrm{NPs}$ in the clusters, and the average particle size was found to be $5,94 \pm 1,48 \mathrm{~nm}$. This is important for the study, as other reports suggest that Pd NPs are ferromagnetic when they reach sizes comparable to or less than $7 \mathrm{~nm}[7,8]$. Therefore, the imaged nanoparticles should be magnetic.

In Fig. 1a, there is no evidence of NPs inside the bacteria. However, this does not mean the absence of small NPs. The resolution of the employed TEM might not be sufficient to visualize them. In fact, there were several reports showing that the High Resolution TEM imaging indeed reveals many small Pd NPs inside the bacteria in similar experiments [12, 13]. These NPs are expected to be magnetic. To confirm this and compare their magnetic properties with those of Pd-Fe and pure $\mathrm{Fe}$ nanoparticle clusters, the MFM study was conducted on slices of resin of the same sample.

\section{B. MFM analysis}

In Fig. 2, the MFM images for one of the samples before the magnetic decoration with $\mathrm{Fe}_{2} \mathrm{O}_{3} \mathrm{NPs}$ are shown. This region of the slice is represented by amplitude (a) and phase shift (b) maps of probe oscillations. The phase shift images was recorded at the lift height of $25 \mathrm{~nm}$ above the measured sample surface. In the amplitude scan (Fig. 2a), it is possible to observe the position of bacteria and the Pd-Fe NPs clusters produced by them. The corresponding phase shift map (Fig. $2 b)$ presents the magnetic regions of the sample. Here, the darker the contrast, the stronger is the magnetic interaction of the sample with the probe, and since the tip is far from the surface, the changes of contrast are produced mainly by magnetic interaction.
The drawback of this phase shift map is that, at the selected lift height, it does not allow to resolve individual NPs. Instead, the spread of the magnetic field lines from individual dipoles and merging of field lines from different nanoparticles create the dark regions, which are the cumulative sum of the stray magnetic fields [27]. Additionally, as Krivcov et al. suggested, an optimal lift height for MFM measurements is also dependent on NP size [22]. In order to resolve NPs of this size in phase shift maps, the tip has to be extremely close to the surface, where the short-range surface forces interfere with the measurements. Although the Pd-based NPs are not visible in the TEM study, the dark regions inside the bacteria are an indication of the magnetic interaction with the tip. It is clear that magnetism inside the bacteria is weaker than in the clusters surrounding them. The strength of the magnetic interaction was quantified measuring histograms of the phase shift, separately in bacteria and in the clusters, and subtracting the phase shift of the background.

It was found that inside bacteria, the mean phase shift value is $-2.70 \pm 0.54$ degrees, while in the clusters outside the bacteria, it is $-16.33 \pm 1.97$ degrees. This suggests that the magnetism of intracellular Pd NPs is about 6 times weaker than it is in the clusters. There is, however, an additional uncertainty in this estimation. The black regions on top of the NP clusters are surrounded by less dark areas, which are most likely coming from the magnetic response of the NPs below the surface of the sample. Their contribution to the magnetism inside the bacteria is not clear. To visualize the magnetism inside of bacteria more clearly, the magnetic decoration technique has been used. The MFM images of a resin slice after magnetic decoration are shown in Fig. 3.

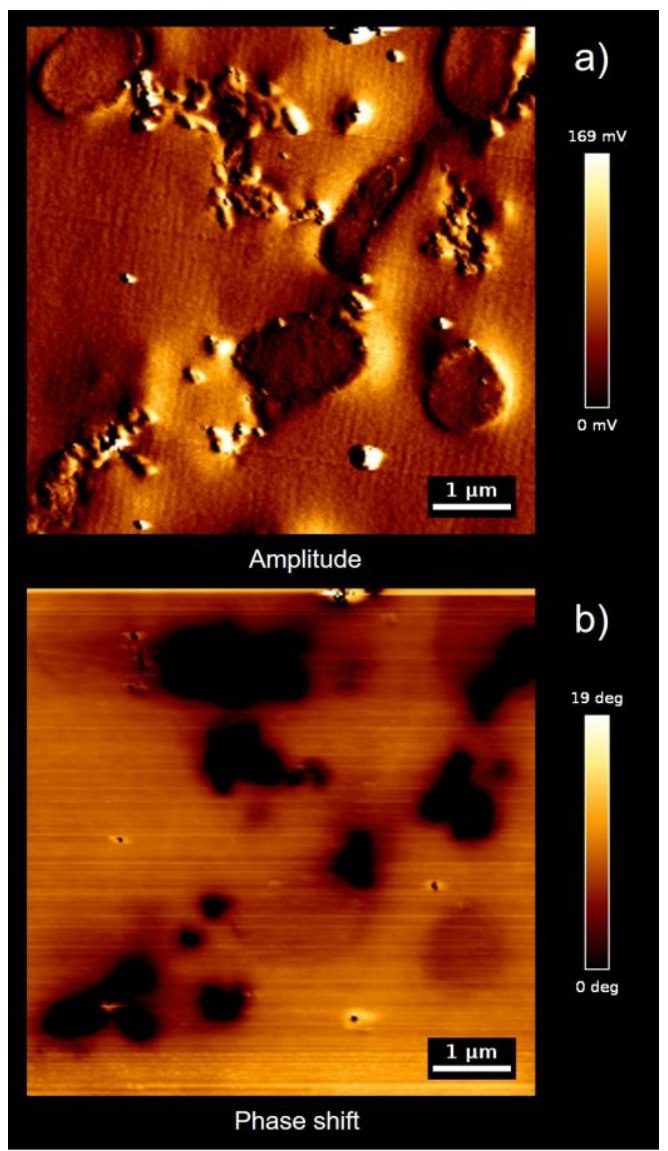

Fig. 2. MFM images of a resin slice with bacteria and clusters of Pd-Fe NPs. (a) Amplitude trace and (b) phase shift retrace maps of the same area 

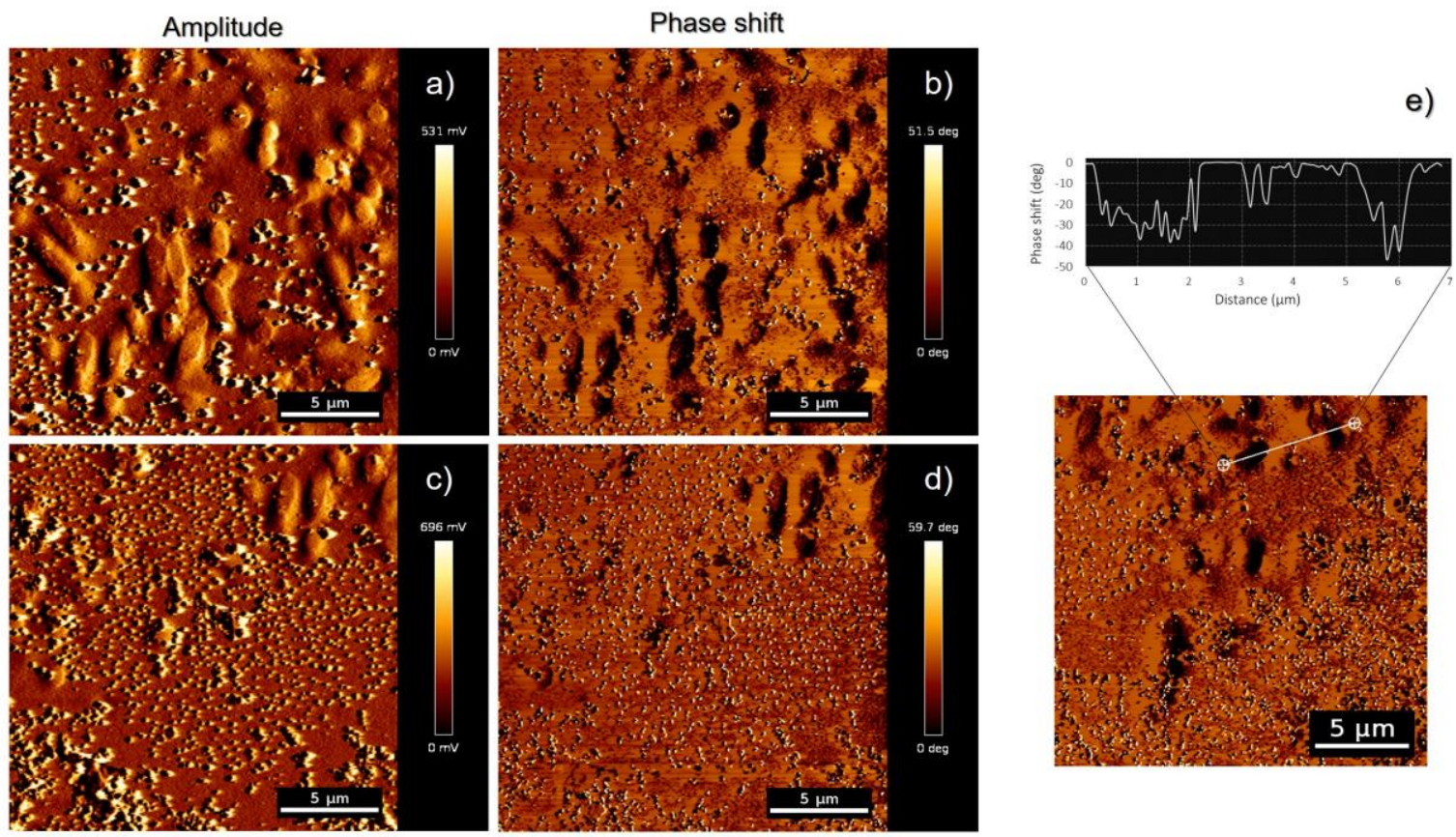

Fig. 3. MFM images of E. coli with Pd-Fe NPs sample after magnetic decoration with $10 \mathrm{~nm} \mathrm{Fe}_{2} \mathrm{O}_{3}$ NPs. Amplitude and phase shift maps of the sample sections with (a-b) high and low (c-d) concentrations of bacteria, respectively. In e), the phase shift line profile for the white line in the lower image, which crosses two Pd NPs-loaded bacteria is shown.

The decoration methodology is similar to Bitter's method [31] used to visualize vortices of magnetic flux in superconductor materials. In this case, ejected magnetic atoms are allowed to settle on a magnetic surface, and it reveals the irregularities in the magnetic flux $[32,33]$. In this work, a drop of aqueous solution of highly magnetic $10 \mathrm{~nm}$ $\mathrm{Fe}_{2} \mathrm{O}_{3}$ NPs was spread on the surface of the resin slice containing bacteria and Pd or Pd-Fe NPs. The deposition was carried out in the presence of a homogeneous external magnetic field, and the $\mathrm{Fe}_{2} \mathrm{O}_{3}$ NPs in the solution move guided by the magnetic force. Due to this, they settle in the places, where they are attracted to other magnetic objects. There is big difference regarding the deposition of the NPs between areas highly covered with bacteria (Figs. 3a-b) and areas free of them (Figs. 3c-d). The non-magnetic areas outside the bacteria appear rough. Similar to other reports [31-33], the $\mathrm{Fe}_{2} \mathrm{O}_{3}$ NPs are accumulated in regions where they group together and form nearly periodically spaced complexes that can be seen as white spots, especially in the amplitude scan in Fig. 3c. We suggest that these regions are formed when the first NPs get attached to the surface forming spots of strong magnetic field, to which the succeeding particles are attracted to. In contrast, the areas above and around bacteria look smooth, as $\mathrm{Fe}_{2} \mathrm{O}_{3}$ NPs spread themselves evenly on top of the weakly-magnetic regions. The scale of the images in Fig. 3 is much bigger than the size of $\mathrm{Fe}_{2} \mathrm{O}_{3}$ NPs, so they cannot be seen individually.

In the phase shift maps in Figs. $3 b$ and $3 d$, it is clearly seen that several bacteria became intensively black, i.e. strongly attracting the oscillating probe. This is the amplification of the magnetic signal, which originally is not strong, as seen in Fig. 2b. The level of amplification can be demonstrated by a line profile across a bacteria. In Fig. 3e, a white line is shown crossing two bacteria, and the phase shift profile is presented. Using the background as reference line, here the phase shift above the bacteria can reach values close to 40-50 degrees. This is about 15 times bigger than original weak response of Pd NPs inside the bacteria and even larger than magnetic response of non-covered Fe and Pd-Fe clusters outside the bacteria. This illustrates the high potential of magnetic decoration as a tool to visualize weak magnetic structures.

\section{CONCLUSIONS}

The use of MFM for the study of weakly-magnetic nanoparticles, such as Pd NPs, is still a growing research field. The limitations set by the sample composition, the magnetic strength, and the particle size, among other, require the development of novel methods and techniques that could help resolving and quantifying their magnetic properties. Although the presence of Pd NPs inside the bacteria could not be confirmed by TEM, the MFM study reveals a weak magnetic response. In this work, a technique similar to Bitter's method was used in order to visualize the weak magnetism of the Pd-based NPs inside the bacteria that produced them. The decoration with external $\mathrm{Fe}_{2} \mathrm{O}_{3}$ nanoparticles produced a strong enhancement of the magnetic signal, up to 15 times higher than the original one. Therefore, the proposed technique, which is frequently used for studies of vortex structures in superconductors, could, in modified form, be useful to study weak magnetic properties of NPs produced within biological samples.

\section{ACKNOWLEDGMENT}

This work is part of the Project "BEDPAN - BioEngineered Palladium Nanoparticles", funded by the Research Council of Norway, RCN294605. The authors would like to thank Associate Professor Irep Gözen and her 
group for providing the iron oxide nanoparticles used in this work. Also, for the support in MFM scans acquisition, Senior Lecturer Anja Røyne is gratefully acknowledged.

\section{REFERENCES}

A. Biffis, P. Centomo, A. Del Zotto, and M. Zecca, "Pd Metal Catalysts for Cross-Couplings and Related Reactions in the 21st Century: A Critical Review," Chemical Reviews, vol. 118, pp. 2249-2295, Feb. 2018.

[2] P. Bera and M. S. Hegde, "Recent advances in auto exhaust catalysis," J. Indian Inst. Sci, vol. 90, pp. 299-325, 2010, Accessed: Apr. 2021

[3] H. Xu, H. Shang, C. Wang, and Y. Du, "Recent Progress of Ultrathin 2D Pd-Based Nanomaterials for Fuel Cell Electrocatalysis," Small, vol. 17, 2005092, Feb. 2021.

[4] K. Hong et al., "Palladium Nanoparticles on Assorted Nanostructured Supports: Applications for Suzuki, Heck, and Sonogashira Cross-Coupling Reactions," ACS Appl. Nano Mater., vol. 3, pp. 2070-2103, Mar. 2020

[5] N. J. Creamer, I. P. Mikheenko, C. Johnson, S. P. Cottrell, and L. E. Macaskie, "Local magnetism in palladium bionanomaterials probed by muon spectroscopy," Biotechnol. Lett., vol. 33, pp. 969-976, Feb. 2011.

[6] M. Nasrollahzadeh, M. Sajjadi, J. Dadashi, and H. Ghafuri, "Pdbased nanoparticles: Plant-assisted biosynthesis, characterization, mechanism, stability, catalytic and antimicrobial activities," Advances in Colloid and Interface Science, vol. 276, 102103, Feb. 2020.

[7] S. Angappane, J. Park, Y. Jang, T. Hyeon, and J. G. Park, "Magnetic Pd nanoparticles: Effects of surface atoms," in Journal of Physics Condensed Matter, vol. 20, 295209, Jul. 2008.

[8] T. Taniyama, E. Ohta, and T. Sato, "Observation of $4 d$ ferromagnetism in free-standing Pd fine particles," Europhys. Lett., vol. 38, pp. 195-200, Mar. 1997.

[9] T. Shinohara, T. Sato, and T. Taniyama, "Surface ferromagnetism of pd fine particles," Phys. Rev. Lett., vol. 91, 197201, Nov. 2003.

[10] X. Xing et al., "Insights into the geometries, electronic and magnetic properties of neutral and charged palladium clusters," Sci. Rep., vol. 6, pp. 1-11, Jan. 2016.

[11] I. M. L. Billas, A. Châtelain, W. A. De Heer, I. M. L. Billas, A. Chatelain, and W. A. De Heer, "Magnetism from the Atom to the Bulk in Iron , Cobalt, and Nickel Clusters," Am. Assoc. Adv. Sci., vol. 265, pp. 1682-1684, Sep. 2017.

[12] J. B. Omajali et al., "Novel catalytically active Pd/Ru bimetallic nanoparticles synthesized by Bacillus benzeovorans," Sci. Rep., vol. 9, pp. 1-12, Mar. 2019.

[13] J. B. Omajali, I. P. Mikheenko, M. L. Merroun, J. Wood, and L. E. Macaskie, "Characterization of intracellular palladium nanoparticles synthesized by Desulfovibrio desulfuricans and Bacillus benzeovorans," J. Nanoparticle Res., vol. 17, pp. 1-17, Jun. 2015.

[14] J. Gomez-Bolivar, I. P. Mikheenko, L. E. Macaskie, and M. L. Merroun, "Characterization of Palladium Nanoparticles Produced by Healthy and Microwave-Injured Cells of Desulfovibrio desulfuricans and Escherichia coli," Nanomaterials, vol. 9, 857, Jun. 2019.

[15] J. M. Foulkes, K. Deplanche, F. Sargent, L. E. Macaskie, and J. R. Lloyd, "A novel aerobic mechanism for reductive palladium biomineralization and recovery by escherichia coli," Geomicrobiol. J., vol. 33, pp. 230-236, Mar. 2016.

[16] D. Chidambaram et al., "Concomitant microbial generation of palladium nanoparticles and hydrogen to immobilize chromate," Environ. Sci. Technol., vol. 44, pp. 7635-7640, Oct. 2010. palladium nanoparticles using origanum vulgare $\mathrm{L}$. extract and their catalytic activity," Molecules, vol. 22, p.165, Jan. 2017.

[18] N. Edayadulla, N. Basavegowda, and Y. R. Lee, "Green synthesis and characterization of palladium nanoparticles and their catalytic performance for the efficient synthesis of biologically interesting di(indolyl)indolin-2-ones,” J. Ind. Eng. Chem., vol. 21, pp. 13651372, Jan. 2015.

[19] J. Claxton, N. Joudeh, A. Royne, D. Linke, and P. Mikheenko, "Sequential Magnetic Mapping of Bacteria Loaded with Pd-Fe Nanoparticles," 2020 IEEE 10th International Conference Nanomaterials: Applications \& Properties (NAP), Sumy, Ukraine, pp01NIC01-1 - 01NIC01-5, Nov. 2020.

[20] A. L. Campaña, N. Joudeh, H. Høyer, A. Røyne, D. Linke, and P. Mikheenko, "Probing van der Waals and magnetic forces in bacteria with magnetic nanoparticles," 2020 IEEE 10th International Conference Nanomaterials: Applications \& Properties (NAP), Sumy, Ukraine, pp. 01NSSA03-1-01NSSA035, Nov. 2020.

[21] K. Deplanche, I. Caldelari, I. P. Mikheenko, F. Sargent, and L. E. Macaskie, "Involvement of hydrogenases in the formation of highly catalytic $\mathrm{Pd}(0)$ nanoparticles by bioreduction of $\mathrm{Pd}(\mathrm{II})$ using Escherichia coli mutant strains," Microbiology, vol. 156, pp. 2630-2640, Sep. 2010.

[22] A. Krivcov, T. Junkers, and H. Möbius, "Understanding electrostatic and magnetic forces in magnetic force microscopy: Towards single superparamagnetic nanoparticle resolution," J. Phys. Commun., vol. 2, 075019, Jul. 2018.

[23] G. Cordova, B. Y. Lee, and Z. Leonenko, "Magnetic force microscopy for nanoparticle characterization," NanoWorld J. vol 2, pp. 10-14, Apr. 2017.

[24] L. Angeloni, D. Passeri, F. A. Scaramuzzo, P. G. Schiavi, F. Pagnanelli, and M. Rossi, "Magnetic force microscopy characterization of core-shell cobalt-oxide/hydroxide nanoparticles," J. Magn. Magn. Mater., vol. 516, 167299, Dec. 2020

[25] B. Torre et al., "Magnetic force microscopy and energy loss imaging of superparamagnetic iron oxide nanoparticles," Sci. Rep., vol. 1, p. 202, Dec. 2011.

[26] I. Safarik, Z. Maderova, K. Pospiskova, K. Horska, and M. Safarikova, "Magnetic decoration and labeling of prokaryotic and eukaryotic cells," RSC Smart Materials, vol. 9, pp. 185-215, Jan. 2014

[27] R. B. Proksch, T. E. Schäffer, B. M. Moskowitz, E. D. Dahlberg, D. A. Bazylinski, and R. B. Frankel, "Magnetic force microscopy of the submicron magnetic assembly in a magnetotactic bacterium," Appl. Phys. Lett., vol. 66, p. 2582, Feb. 1995.

[28] C. Marcuello, L. Chambel, M. S. Rodrigues, L. P. Ferreira, and M. M. Cruz, "Magnetotactic Bacteria: Magnetism beyond Magnetosomes," IEEE Trans. Nanobioscience, vol. 17, pp. 555559, Oct. 2018

[29] Y. Chao and T. Zhang, "Optimization of fixation methods for observation of bacterial cell morphology and surface ultrastructures by atomic force microscopy," Appl. Microbiol. Biotechnol., vol. 92, pp. 381-392, Oct. 2011.

[30] J. I. Goldstein et al., Scanning Electron Microscopy and X-ray Microanalysis. Boston, MA: Springer US, 2003.

[31] F. Bitter, "On inhomogeneities in the magnetization of ferromagnetic materials," Phys. Rev., vol. 38, pp. 1903-1905, Nov. 1931

[32] L. Y. Vinnikov, I. S. Veshchunov, M. S. Sidelnikov, and V. S. Stolyarov, "The High-Resolution Bitter Decoration Technique for the Magnetic Flux Structure Imaging at Low Temperatures," Instruments Exp. Tech., vol. 62, pp. 587-593, Aug. 2019.

[33] T. Tamegai, H. Aoki, M. Matsui, and M. Tokunaga, "Bitter decoration and magneto-optical observations of vortex chains in high temperature superconductors," Pramana - J. Phys., vol. 66, pp. 271-278, Jan. 2006. 